\title{
PTH-023 APPROPRIATE STAGING RENDERS ABLATIVE THERAPY SAFE FOR HIGH GRADE DYSPLASIA IN BARRETT'S OESOPHAGUS
}

doi:10.1136/gut.2011.239301.424

P Sellaturay, ${ }^{1, *}$ R Haidry, J Dunn, ${ }^{1}$ S Green, ${ }^{1}$ M Rodriguez-Justo, ${ }^{1}$ A Winstanley, M Novelli, ${ }^{1}$ M Banks, ${ }^{1}$ L Lovat' ${ }^{1}$ UCLH, London, UK

Introduction NICE approval of ablative therapy for high grade dysplasia (HGD) in Barrett's oesophagus (BE) means more patients will want this treatment. Concerns remain regarding the risk of missing cancer at initial staging and the reliability of detecting dysplasia at repeat endoscopies. It is not clear whether dysplasia recurring after ablation is at the same or different locations.

Aims To assess the likelihood of cancer and reliability of detecting dysplasia prior to therapy and the site of recurrence of dysplasia after ablation.

Methods 64 patients with HGD arising in BE were entered into a randomised controlled trial of Photofrin $(n=29)$ versus ALA ( $n=31)$ photodynamic therapy between February 2006 and March 2010. All patients underwent 2 staging endoscopies prior to initial therapy with endoscopic ultrasound and quadrantic biopsies taken every $2 \mathrm{~cm}$ throughout the BE segment. These procedures were carried out $2-3$ months apart. Presence of HGD was confirmed by 2 independent specialist GI pathologists. Endoscopic follow-up after PDT was at $3,6,12,18,24$ months and yearly thereafter. Residual or recurrent $\mathrm{HGD}$ was retreated with $\mathrm{PDT}$ and follow-up continued as before. Comparison was made between original biopsies and those taken at follow-up endoscopy. Any site within $2 \mathrm{~cm}$ of the original biopsy was considered as the same location.

Results 4 patients (6\%) were withdrawn prior to therapy as they developed invasive cancer. Of the remaining 60, 32 (53\%) had a single PDT, 19 (32\%) had 2 treatments and $9(15 \%)$ had 3 PDT sessions. 11 patients (18\%) had EMR prior to first PDT and a further $3(5 \%)$ required EMR between PDT treatments for new nodules. Although dysplasia is supposed to be patchy and the authors only took $2 \mathrm{~cm}$ quadrantic biopsies, the authors still found the dysplasia at the second endoscopy in $85 \%$ of patients with no difference between unifocal and multifocal dysplasia. When patients needed more than a single treatment, over half had less extensive dysplasia than at baseline and this had better final outcome. When dysplasia recurred after PDT, 
it was at the same location in $50 \%$ of cases and in the distal oesophagus in the other $50 \%$.

Conclusion After accurate staging and repeat endoscopy, less than $10 \%$ of patients were shown to have invasive cancer. The authors were also able to accurately locate dysplasia at a second endoscopy, even when it was apparently unifocal. Repeat treatments were frequently needed and a marker of likely success was the decreasing number of areas of dysplasia remaining after each treatment. Metachronous lesions tended to occur at the original site of dysplasia or in the distal oesophagus, in line with evidence published by other groups. This findings offer reassurance that first line ablative therapy is a safe option.

\section{Competing interests None.}

